\title{
Entrevista Motivacional para Estudantes da Área da Saúde: Resultados de um Treinamento*
}

\author{
Fernanda Pasquoto de Souza ${ }^{1, * *}$, Elisabeth Meyer $^{2}$, \& Margareth da Silva Oliveira ${ }^{3}$ \\ ${ }^{1}$ Universidade Luterana do Brasil, Canoas, RS, Brasil \\ ${ }^{2}$ Universidade Federal do Rio Grande do Sul, Porto Alegre, RS, Brasil \\ ${ }^{3}$ Pontifícia Universidade Católica do Rio Grande do Sul, Porto Alegre, RS, Brasil
}

\begin{abstract}
RESUMO - Este estudo procurou avaliar a efetividade de um treinamento em Entrevista Motivacional (EM) para estudantes do último ano de diferentes cursos na área da saúde. Usando um delineamento pré e pós-teste quase-experimental, 60 estudantes receberam um treinamento de oito horas de habilidades básicas da Entrevista Motivacional. As habilidades foram avaliadas por meio de uma vinheta clínica, das Réguas de Confiança e Importância e do Questionário de Resposta Útil, codificado utilizando o Motivational Interviewing Skill Code. O treinamento foi associado com melhora no uso das habilidades básicas da Entrevista Motivacional. Os alunos também aumentaram a percepção da importância em utilizar essas habilidades com os pacientes, mesmo com um treinamento breve.
\end{abstract}

PALAVRAS-CHAVE: estudantes de graduação, entrevista motivacional, treinamento, cursos da saúde

\section{Motivational Interviewing for Health Sciences Students: The Results of Training}

\begin{abstract}
This study sought to evaluate the effectiveness of Motivational Interviewing (MI) training on undergraduate students in the senior year of different healthcare courses. Using a pre and post-test quasi-experimental design, 60 undergraduate students received an eight-hour Motivational Interviewing basic skills training. Basic skills were assessed using a clinical vignette, the Confidence and Importance Ruler and The Helpful Responses Questionnaire, which was coded using the Motivational Interviewing Skill Code. The training was associated with improvements in motivational interviewing basic skills. Students also showed increased awareness of the importance of using these skills with patients, even after a brief training.
\end{abstract}

KEYWORDS: graduate students, motivational interviewing, training, health courses

A Entrevista Motivacional (EM) é uma abordagem de tratamento que auxilia o indivíduo no reconhecimento de um problema e possíveis ambivalências para a mudança de comportamentos. Sendo assim, é um meio de comunicação colaborativa entre profissional da saúde e paciente que propicia um ambiente favorável para que o sujeito possa identificar suas motivações intrínsecas e promover melhores resultados no tratamento (Miller \& Rollnick, 2013; Dray \& Wade, 2012). É orientada para metas com atenção especial à Conversa sobre Mudança, que é qualquer fala do paciente que o mova em direção à mudança, sendo planejada para reforçar a motivação pessoal e o compromisso com um comportamento-alvo. Para tanto, o profissional da saúde evoca e explora as próprias razões do paciente para a mudança, dentro de uma atmosfera de aceitação e empatia, e pode ser aplicado nas diferentes especialidades da saúde (Lundahl \& Burke, 2009).

Os benefícios do treinamento da EM com diferentes profissionais da saúde podem ser verificados em revisões sistemáticas (Madson, Loignon, \& Lane, 2009; Söderlund, Madson, Rubak, \& Nilsen, 2011). Esses estudos permitiram concluir que há fortes relações positivas entre as habilidades

\footnotetext{
* Apoio Pró-Bolsas PUC/RS

** E-mail: fegps@terra.com.br

- Submetido: 16/05/2015; Revisado: 07/06/2016; Aceito: 12/08/2016.
} 
de comunicação do profissional da saúde e a capacidade do paciente em adotar comportamentos de saúde mais saudáveis e/ou preventivos. Dessa forma, a competência do profissional da saúde para explicar, ouvir e ser empático pode ter um efeito profundo sobre os resultados do tratamento, bem como a satisfação do paciente com seu autocuidado.

Os alunos de graduação das diferentes áreas da saúde são treinados para adquirir habilidades e práticas profissionais muito distintas. No entanto, nenhum curso de graduação no Brasil oferece rotineiramente treinamento de EM apesar da sua eficácia ser amplamente reconhecida.
Mais do que isso, nas diferentes graduações da área da saúde, nenhuma estratégia específica é ensinada a respeito de como o profissional pode ajudar um paciente a lidar com a ambivalência em relação a mudar um determinado comportamento não saudável.

Até onde sabemos, no Brasil nenhum treinamento específico de EM é oferecido regularmente durante o curso de graduação nas diferentes áreas da saúde. O objetivo deste estudo é avaliar um treinamento básico de EM para estudantes no último ano de graduação, em diferentes cursos na área da saúde, em uma Universidade Privada.

\section{MÉTODO}

Usando um delineamento pré e pós-teste quaseexperimental, 60 estudantes do último ano da graduação de Medicina, Fisioterapia, Odontologia, Psicologia, Enfermagem e Nutrição da Pontifícia Universidade Católica do Rio Grande do Sul (PUC/RS) receberam um treinamento de oito horas em EM. Após aprovação do projeto de pesquisa pelo Comitê de Ética em Pesquisa envolvendo seres humanos (CAAE - 18995613.2.0000.5336), foram efetuados contatos com os professores dos diferentes cursos, com o objetivo de apresentar o estudo e solicitar a colaboração dos mesmos para a divulgação em sala de aula. Os alunos foram informados pelos professores sobre os objetivos, dias de treinamento disponíveis e local, não sendo necessário fazer qualquer tipo de inscrição ou préagendamento. É importante ressaltar que foi avisado que só poderiam participar aqueles alunos que não tiveram qualquer treinamento formal de EM. O Termo de Consentimento Livre e Esclarecido da Pesquisa (TCLE) foi entregue no dia do treinamento, antes do mesmo iniciar.

Diversas variáveis podem ser utilizadas como indicadores de conhecimento em EM. O protocolo para a avaliação foi composto de: (1) uma vinheta retirada do livro de Rollnick, Miller e Butler (2009), em que deveriam identificar habilidades básicas da EM: Pergunta Aberta, Afirmação, Reflexão e Resumo, além de uma fala do paciente que demonstrasse Resistência à mudança do comportamento, sendo pontuada como acertou ou errou; (2) como sugerido por Miller e Rollnick (2002), a Régua de Importância (quão importante diria que é aprender sobre EM em uma escala de 0-10) e a Régua de Confiança (quão confiante diria que está, se decidir usar a EM em seus atendimentos, de que poderia ter sucesso em uma escala de 0-10) e (3) o Questionário de Resposta Útil (Helpful Responses Questionnaire; Miller, Hedrick, \& Orlofsky, 1991), que apresenta seis hipotéticas declarações de pacientes e pede ao participante para escrever "o que diria em seguida", em resposta a cada declaração, com o objetivo de avaliar até que ponto o indivíduo utilizou Pergunta Aberta, Afirmação, Reflexão, Resumo, Empatia, Aconselhou, Pergunta Fechada ou foi Julgamental.

Esse questionário é uma alternativa quando a observação individual não é possível, como quando os indivíduos estão sendo avaliados em um treinamento. No Questionário de Resposta Útil, foi utilizada uma parte dos indicadores de habilidades em EM, conforme indicado pelo instrumento Motivational Interviewing Skill Code (MISC; Miller, Moyers, Ernst, \& Amrhein, 2008). O MISC, segundo seus autores, é utilizado para avaliar a eficácia do treinamento em EM, comparando as habilidades do profissional antes e depois do treino. Para tanto, mede os comportamentos do profissional em relação ao percentual de Perguntas Abertas e Fechadas, assim como se é Consistente com EM (essa categoria é usada para observar determinados comportamentos do profissional que são consistentes com a abordagem da EM: Pergunta Aberta, Empatia, Afirmação, Reflexão, Resumo) ou Inconsistente com EM (Pergunta Fechada, Aconselhar, Julgar ou Confrontar).

A Pergunta Aberta permite uma ampla gama de possíveis respostas. A Empatia se destina a captar o quanto o profissional entende e/ou faz um esforço para compreender com precisão a perspectiva do paciente. A Afirmação se diz algo positivo ou que estimula o paciente a manter determinado comportamento durante a consulta. A Reflexão envolve capturar e devolver em resposta a uma declaração do paciente. O Resumo reúne tudo que foi dito, permitindo a transição para o próximo tópico. A Pergunta Fechada quando implica uma resposta curta: sim ou não, um número, etc. Aconselhar é identificado quando dá conselhos, faz uma sugestão ou oferece uma solução ou possível ação. Julgar diz respeito a avaliar a fala do paciente de forma condenatória. Confrontar aparece quando estabelece uma relação de poder desigual, acompanhada de uma fala que desaprova, discorda ou questiona a honestidade do paciente. Caso o participante escrevesse mais de uma frase para a questão, somente a primeira era considerada na análise.

Esses questionários foram distribuídos antes e logo após o término do treinamento. Para garantir a confidencialidade, nenhum nome apareceu no protocolo. Cada participante recebeu um número e esse código permitiu comparar os instrumentos pré e pós-treinamento. As respostas à vinheta e ao Questionário de Resposta Útil foram aferidas individualmente por três avaliadores independentes, cegados para o curso dos participantes, sendo todos $\mathrm{PhD}$ em 
Psicologia e com treinamento avançado em EM no Squaretop (Albuquerque, USA). As três avaliações foram comparadas entre si, para efeitos de confiabilidade intrajuízes.

O formato do treinamento foi de workshop de um dia, com duração de 8 horas. O treinamento contemplou: (1) o Espírito da EM; (2) as habilidades de aconselhamento centradas no paciente; (3) reconhecer e reforçar a "conversa sobre mudança"; (4) provocar e fortalecer a "conversa sobre mudança" e (5) acompanhar a resistência. O workshop foi conduzido por um dos membros da equipe do estudo, que é $\mathrm{PhD}$ em psiquiatria, com larga experiência na realização de oficinas de capacitação em EM para diferentes áreas da saúde. $\mathrm{O}$ treinamento incluiu apresentações didáticas e exercícios vivenciais, como recomendado por Moyers, Martin, Manuel, Miller e Ernst (2007). Os exercícios vivenciais, como roleplay, ocuparam $70 \%$ do tempo total do treinamento, sendo realizados em duplas e, eventualmente, com um terceiro participante como observador. O instrutor forneceu feedback sobre os exercícios ao longo do treinamento.

\section{Análise dos Dados}

A análise dos dados foi realizada no programa SPSS, versão 21.0. As variáveis quantitativas foram descritas por média e desvio padrão e as qualitativas por frequências absolutas e relativas. Para fins de comparação, foram utilizadas as fórmulas do percentual de acertos na vinheta, calculado sob a fórmula: $\mathrm{n}^{\mathrm{o}}$ de acertos nos cinco comportamentos/total de comportamentos x 100. Também foram calculados os percentuais de perguntas fechadas e abertas para as seis questões do Questionário de Resposta Útil, da seguinte forma: $\mathrm{n}^{\circ}$ de questões em que foi utilizada a Pergunta Aberta ou Fechada/total de questões x 100. Foi calculado também o percentual Consistente com EM, através da seguinte fórmula: número de questões em que foi utilizado comportamentos Consistente com EM/total de questões $\mathrm{x} 100$.

O mesmo raciocínio foi empregado para o cálculo do percentual Inconsistente com EM. Foi utilizada a Análise de Variância (ANOVA) one-way, complementada pelo teste de Tukey para comparar as médias entre os grupos. Nas comparações antes e após o treinamento, os testes de McNemar (variáveis categóricas) e $t$-student pareado (variáveis quantitativas) foram empregados. Foi usada a medida de Cohen para avaliar o efeito do treinamento. A interpretação mais utilizada é de que se o tamanho de efeito padronizado for inferior a 0,5 , ele é considerado pequeno, entre 0,5 e 0,79 é considerado moderado e igual ou acima de 0,8 é considerado grande (Cohen, 1998). O nível de significância adotado foi de $5 \%(\mathrm{p} \leq 0,05)$.

\section{RESULTADOS}

A amostra foi composta por 60 alunos, com média de idade de 25,7 anos $( \pm 7,5)$, variando de 18 a 55 anos. A predominância foi do sexo feminino $(90 \%)$ e cada curso contou com 10 alunos (16,7\%). O kappa entre os juízes mostrou uma concordância substancial, sendo de 0,78 para o juiz 1 versus o juiz 2, 0,82 entre os juízes 1 e 3 e 0,95 entre os juízes 2 e 3 .

Análises exploratórias demonstraram que o treinamento de oito horas foi suficiente para aumentar a habilidade com a EM, conforme apresenta a Tabela 1. Foi possível observar, no total da amostra, que cinco dos dez indicadores que avaliam a retenção do conhecimento sobre EM mudaram de forma significativa. A média de diferenças nos cinco indicadores entre o pré/pós-treinamento apresentaram, em sua maioria $(60 \%)$, efeito grande $(E S>0,8)$, variando de 0,33 para 1,57 , em todos os indicadores.

Em relação ao Questionário de Resposta Útil, os participantes apresentaram um aumento em ser Consistente com EM e, consequentemente, diminuição das Perguntas Fechadas e ser Inconsistente com a EM. Finalmente, o tamanho de efeito observado demonstra que teve maior impacto o Questionário de Resposta Útil e menor impacto nas Réguas, em especial na Régua de Confiança. A Tabela 1 permite observar a comparação dos desfechos pré e póstreinamento na amostra total.
Tabela 1

Comparação dos Desfechos Pré e Pós Treinamento

\begin{tabular}{lcccc}
\hline Variáveis & Pré (n=60) & Pós (n=60) & p & ES \\
\hline $\begin{array}{l}\text { Vinheta } \\
\text { (número de acertos) }\end{array}$ & & & & \\
Pergunta Aberta & $35(58,3)$ & $44(73,3)$ & $0,150^{*}$ & - \\
Afirmação & $20(33,3)$ & $48(80,0)$ & $<0,001^{*}$ & - \\
Resumo & $25(41,7)$ & $36(60,0)$ & $0,061^{*}$ & - \\
Escuta Reflexiva & $23(38,3)$ & $23(38,3)$ & $1,000^{*}$ & - \\
$\begin{array}{l}\text { Resistência } \\
\text { Percentual de acertos }\end{array}$ & $23(38,3)$ & $31(51,7)$ & $0,134^{*}$ & - \\
total & $42,0 \pm 22,3$ & $60,7 \pm 20,5$ & $<0,001^{* *}$ & 0,87 \\
Questionário & & & & \\
de resposta útil & & & & \\
$\begin{array}{l}\text { Percentual de } \\
\text { Perguntas Abertas }\end{array}$ & $26,1 \pm 24,4$ & $35,0 \pm 29,9$ & $0,106^{* *}$ & 0,33 \\
$\begin{array}{l}\text { Percentual de } \\
\text { Perguntas Fechadas }\end{array}$ & $14,4 \pm 18,5$ & $4,72 \pm 9,75$ & $<0,001^{* *}$ & 0,65 \\
Consistente com EM & $44,4 \pm 33,3$ & $87,8 \pm 20,3$ & $<0,001^{* *}$ & 1,57 \\
$\begin{array}{l}\text { Inconsistente } \\
\text { com EM }\end{array}$ & $55,6 \pm 33,3$ & $12,2 \pm 20,3$ & $<0,001^{* *}$ & 1,57 \\
Régua Importância & $6,80 \pm 2,72$ & $8,45 \pm 1,38$ & $<0,001^{* *}$ & 0,77 \\
Régua Confiança & $6,90 \pm 2,97$ & $7,47 \pm 1,97$ & $0,161^{* *}$ & 0,24 \\
\hline
\end{tabular}

EM=Entrevista Motivacional; ES=Effect Size by Cohen * Teste de McNemar; ** Teste t-student pareado 
Quando avaliado por curso, somente no curso de Medicina não houve uma melhora significativa no percentual de comportamentos Consistentes com EM. O percentual de acertos na vinheta foi significativamente mais elevado após o treinamento nos cursos de Odontologia, Enfermagem e Fisioterapia. Na Tabela 2, são apresentadas as mudanças obtidas pelos participantes nos diversos instrumentos pré e pós-treino.

Tabela 2

Comparação entre os Cursos em Média e Desvio Padrão

\begin{tabular}{|c|c|c|c|c|}
\hline Cursos & Pré $(n=10)$ & Pós $(n=10)$ & $\mathbf{P}$ & ES \\
\hline \multicolumn{5}{|l|}{ Psicologia } \\
\hline Vinheta - Percentual de acertos total & $46,0 \pm 26,7$ & $60,0 \pm 21,1$ & 0,089 & 0,58 \\
\hline Questionário de Resposta Útil - Consistente com EM & $56,7 \pm 23,8$ & $95,0 \pm 8,1$ & $<0,001$ & 2,15 \\
\hline Questionário de Resposta Útil - Percentual Perguntas Abertas & $26,7 \pm 19,6$ & $15,0 \pm 20,0$ & 0,226 & 0,59 \\
\hline Questionário de Resposta Útil - Percentual Perguntas Fechadas & $21,7 \pm 26,1$ & $5,0 \pm 8,1$ & 0,063 & 0,86 \\
\hline Régua Importância & $7,50 \pm 3,17$ & $8,50 \pm 2,01$ & 0,353 & 0,38 \\
\hline Régua Confiança & $6,70 \pm 2,95$ & $8,00 \pm 1,87$ & 0,258 & 0,53 \\
\hline \multicolumn{5}{|l|}{ Enfermagem } \\
\hline Vinheta - Percentual de acertos total & $40,0 \pm 21,1$ & $60,0 \pm 24,9$ & 0,008 & 0,87 \\
\hline Questionário de Resposta Útil - Consistente com EM & $41,7 \pm 23,9$ & $98,3 \pm 5,3$ & $<0,001$ & 3,27 \\
\hline Questionário de Resposta Útil - Percentual Perguntas Abertas & $30,0 \pm 23,3$ & $31,7 \pm 14,6$ & 0,859 & 0,09 \\
\hline Questionário de Resposta Útil - Percentual Perguntas Fechadas & $8,33 \pm 11,8$ & $0,00 \pm 0,00$ & 0,052 & 1,00 \\
\hline Régua Importância & $5,60 \pm 3,17$ & $7,70 \pm 0,95$ & 0,059 & 0,90 \\
\hline Régua Confiança & $9,20 \pm 0,79$ & $9,30 \pm 0,68$ & 0,811 & 0,14 \\
\hline \multicolumn{5}{|l|}{ Nutrição } \\
\hline Vinheta - Percentual de acertos total & $56,0 \pm 22,7$ & $64,0 \pm 18,4$ & 0,423 & 0,39 \\
\hline Questionário de Resposta Útil - Consistente com EM & $48,3 \pm 29,9$ & $90,0 \pm 17,9$ & 0,002 & 1,69 \\
\hline Questionário de Resposta Útil - Percentual Perguntas Abertas & $41,7 \pm 31,7$ & $21,7 \pm 31,5$ & 0,211 & 0,63 \\
\hline Questionário de Resposta Útil - Percentual Perguntas Fechadas & $13,3 \pm 15,3$ & $6,7 \pm 11,7$ & 0,309 & 0,48 \\
\hline Régua Importância & $8,70 \pm 2,00$ & $8,90 \pm 1,37$ & 0,619 & 0,12 \\
\hline Régua Confiança & $5,80 \pm 2,44$ & $6,70 \pm 1,64$ & 0,337 & 0,43 \\
\hline \multicolumn{5}{|l|}{ Odontologia } \\
\hline Vinheta - Percentual de acertos total & $34,0 \pm 21,2$ & $68,0 \pm 21,5$ & 0,003 & 1,59 \\
\hline Questionário de Resposta Útil - Consistente com EM & $10,0 \pm 16,1$ & $86,7 \pm 17,2$ & $<0,001$ & 4,60 \\
\hline Questionário de Resposta Útil - Percentual Perguntas Abertas & $8,33 \pm 16,2$ & $45,0 \pm 34,3$ & 0,022 & 1,37 \\
\hline Questionário de Resposta Útil - Percentual Perguntas Fechadas & $20,0 \pm 24,6$ & $3,3 \pm 10,5$ & 0,023 & 0,88 \\
\hline Régua Importância & $7,40 \pm 1,71$ & $9,10 \pm 0,74$ & 0,003 & 1,29 \\
\hline Régua Confiança & $4,30 \pm 2,67$ & $6,90 \pm 1,20$ & 0,019 & 1,26 \\
\hline \multicolumn{5}{|l|}{ Medicina } \\
\hline Vinheta - Percentual de acertos total & $42,0 \pm 25,7$ & $62,0 \pm 17,5$ & 0,052 & 0,91 \\
\hline Questionário de Resposta Útil - Consistente com EM & $73,3 \pm 41,7$ & $68,3 \pm 34,6$ & 0,820 & 0,13 \\
\hline Questionário de Resposta Útil - Percentual Perguntas Abertas & $28,3 \pm 20,9$ & $55,0 \pm 31,5$ & 0,091 & 1,00 \\
\hline Questionário de Resposta Útil - Percentual Perguntas Fechadas & $8,33 \pm 14,2$ & $11,7 \pm 13,7$ & 0,662 & 0,24 \\
\hline Régua Importância & $5,80 \pm 1,40$ & $8,80 \pm 0,79$ & $<0,001$ & 2,64 \\
\hline Régua Confiança & $7,10 \pm 3,32$ & $5,40 \pm 2,27$ & 0,237 & 0,60 \\
\hline \multicolumn{5}{|l|}{ Fisioterapia } \\
\hline Vinheta - Percentual de acertos total & $34,0 \pm 9,7$ & $50,0 \pm 19,4$ & 0,022 & 1,04 \\
\hline Questionário de Resposta Útil - Consistente com EM & $36,7 \pm 27,0$ & $88,3 \pm 13,7$ & $<0,001$ & 2,41 \\
\hline Questionário de Resposta Útil - Percentual Perguntas Abertas & $21,7 \pm 24,9$ & $41,7 \pm 28,6$ & 0,089 & 0,75 \\
\hline Questionário de Resposta Útil - Percentual Perguntas Fechadas & $15,0 \pm 14,6$ & $1,67 \pm 5,27$ & 0,022 & 1,21 \\
\hline Régua Importância & $5,80 \pm 3,26$ & $7,70 \pm 1,57$ & 0,070 & 0,74 \\
\hline Régua Confiança & $8,30 \pm 2,71$ & $8,50 \pm 1,08$ & 0,758 & 0,10 \\
\hline
\end{tabular}


$\mathrm{Na}$ comparação entre os cursos, houve diferença significativa quanto à variação na Régua de Confiança $[F(5,54)=2,40 ; p=0,049]$, sendo que os alunos do curso de Odontologia apresentaram um aumento mais acentuado na confiança com a EM, quando comparado com os alunos do curso de Medicina. Para os demais cursos, as diferenças não foram significativas (Figura 1).

Também houve diferença significativa entre os cursos quanto à variação média no percentual de respostas compatíveis com a EM após o treinamento $[\mathrm{F}(5,54)=5,64$; $\mathrm{p}<0,001]$, sendo que os alunos do curso de Odontologia, Enfermagem e Fisioterapia apresentaram um aumento mais acentuado no percentual de respostas Consistentes com EM, quando comparado com os alunos do curso de Medicina. Para os demais cursos, as diferenças não foram significativas como pode ser visto na Figura 2.

Por fim, houve diferença significativa entre os cursos quanto ao percentual de Perguntas Abertas do Questionário de Resposta Útil após o treinamento $[\mathrm{F}(5,54)=3,47$; $\mathrm{p}=0,009]$, sendo que os alunos do curso de Odontologia apresentaram um aumento mais acentuado nessa variável quando comparados com os alunos do curso de Nutrição. Para os demais cursos, as diferenças não foram significativas (Figura 3).

Não foi observada mudança significativa nos níveis de confiança em utilizar as habilidades da EM após o treinamento. É importante destacar que 19 alunos $(31,7 \%)$ diminuíram a confiança em utilizar a EM com os pacientes. Além disso, quando comparadas às mudanças nas variáveis após o treinamento com o sexo e a idade do aluno, as diferenças não foram significativas.

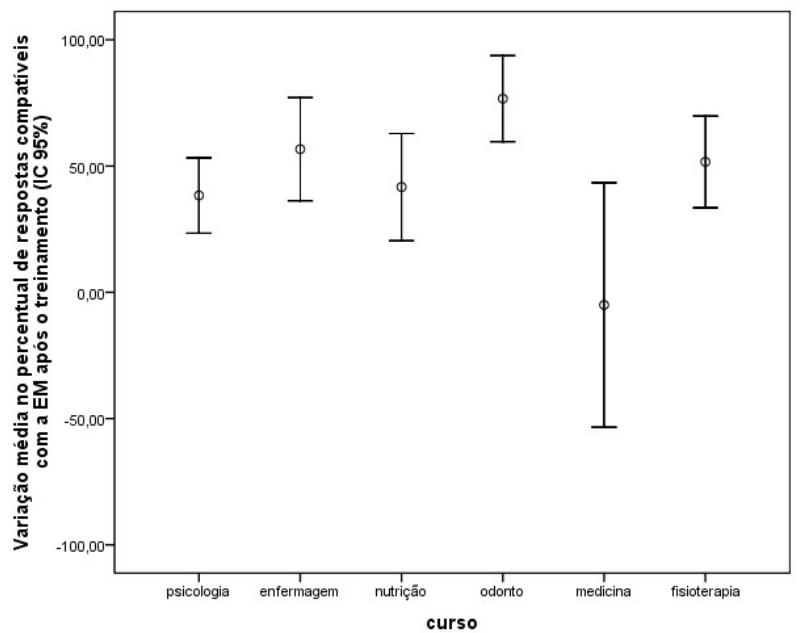

a,b Letras iguais não diferem pelo teste de Tukey a 5\% de significância Figura 2. Variação média no percentual de respostas Consistente com a Entrevista Motivacional (EM) após o treinamento conforme o curso. Os círculos representam a média e as barras de erro os limites inferior e superior do intervalo de $95 \%$ de confiança.

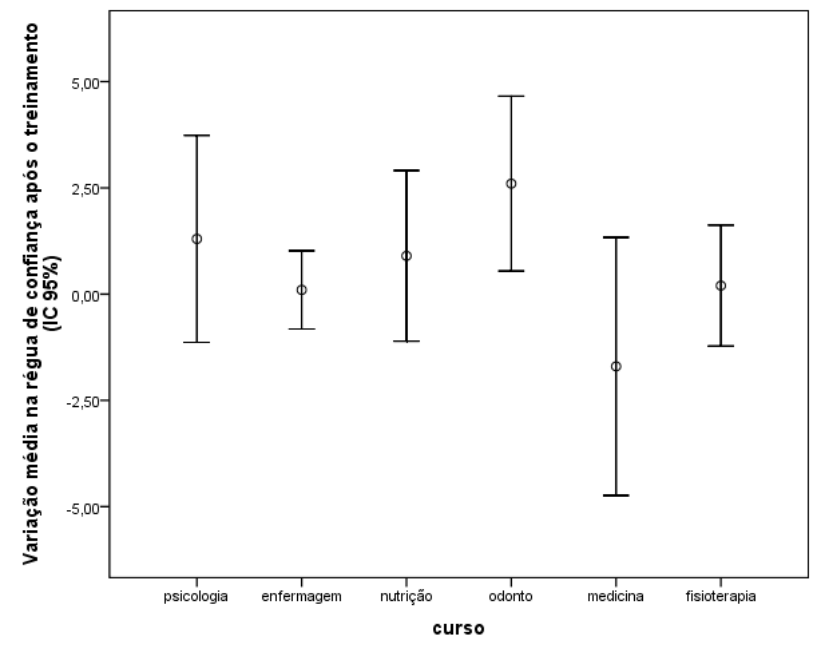

a,b Letras iguais não diferem pelo teste de Tukey a 5\% de significância Figura 1. Variação média na Régua de Confiança após o treinamento conforme o curso. Os círculos representam a média e as barras de erro os limites inferior e superior do intervalo de $95 \%$ de confiança.

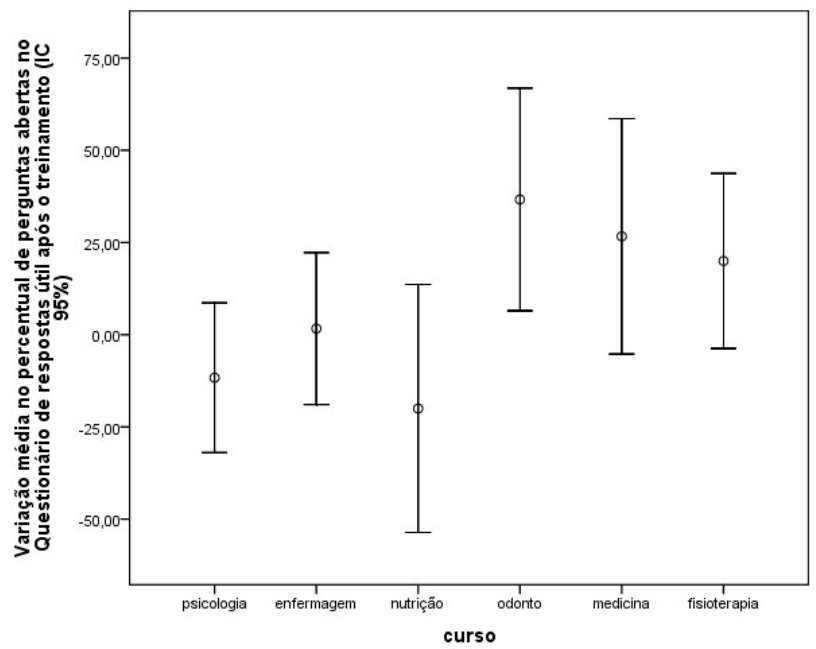

${ }^{\mathrm{a}, \mathrm{b}}$ Letras iguais não diferem pelo teste de Tukey a 5\% de significância Figura 3. Variação média no percentual de Perguntas Abertas do Questionário de Resposta Útil após o treinamento conforme o curso. Os círculos representam a média e as barras de erro os limites inferior e superior do intervalo de $95 \%$ de confiança 


\section{DISCUSSÃO}

Estabelecer um treinamento de oito horas de duração de EM para estudantes de graduação na área da saúde já foi descrito em outros estudos (Bell \& Cole, 2008; Daeppen et al., 2012). A avaliação pré-treinamento permitiu observar que um curso não possuía maior conhecimento das habilidades básicas da EM do que outro. Os resultados dos questionários pré e pós-treinamento indicaram que $o$ treinamento melhorou a capacidade dos alunos em usar a EM, como estimado pelos avaliadores independentes, embora algumas das habilidades mais sutis da EM foram mais difíceis de dominar em um tempo tão curto. Ainda, os estudantes acreditavam que aprender as habilidades da EM é importante e que o treinamento melhorou a sua confiança em utilizá-la.

Em relação aos cursos não terem tido um aumento significativo na Régua de Confiança, a melhora mais acentuada no grupo de alunos da Odontologia pode ser possivelmente explicada pelo baixo nível de confiança que os alunos tinham na EM antes do treinamento. Além disso, uma possível justificativa para a acentuada melhora no grupo de alunos da Odontologia pode ser pelo baixo percentual de Perguntas Abertas realizadas no Questionário de Resposta Útil antes do treinamento. A EM não é fácil, porque requer muito treinamento, o que já foi assinalado por Miller e Rollnick (2009).

Os alunos dos cursos de Medicina, Psicologia e Nutrição apresentaram médias de percentual dos itens relacionados à EM mais elevados no pré-treinamento, quando comparados com os da Odontologia. Dessa forma, parece que o grupo que mais se beneficiou do treinamento foi o dos alunos da Odontologia, seguidos pelos da Enfermagem e Fisioterapia, principalmente quando comparados aos alunos do curso de Medicina. Uma provável explicação em relação ao fato dos alunos da Medicina não terem aumento no percentual de respostas Consistentes com a EM pode ser que, antes do treinamento, esse percentual já era mais elevado do que nos demais cursos. Embora aparentemente importante em termos de formação, as características de cada curso de graduação não parecem ser centrais para a relação entre o curso e os resultados do treinamento. No entanto, os presentes dados não podem explicar esse resultado e essa questão deve ser abordada em pesquisas futuras.

Algumas limitações devem ser assinaladas. Primeira, embora regularmente empregado, é relevante observar que os limiares de proficiência para os Resumos, Empatia e Resistência são baseados na opinião dos avaliadores e, conforme Moyers et al., (2007), no momento não existe nenhum dado de validade para apoiá-los. Segunda, quantificar as respostas dos participantes permitiu comparar as avaliações pré/pós-treinamento e não teve como objetivo avaliar a qualidade das respostas, mas simplesmente contá-las. Terceira, tendo em conta que o nosso período de treinamento foi limitado a medir diferenças apenas antes e logo após a atividade, isto restringe a nossa capacidade de avaliar a melhoria e manutenção dos resultados em longo prazo. Estudos de seguimento com essa amostra são importantes, pois permitirão avaliar a manutenção dos ganhos dessas habilidades, o impacto do treinamento sobre o comportamento dos estudantes na prática clínica no mundo real, bem como a influência nos resultados clínicos. Quarta, um delineamento do tipo quasi-experimento sem qualquer tipo de grupo controle impede comparar as habilidades adquiridas no treinamento versus estudantes não treinados.

Por fim, esses resultados refletem os efeitos do treinamento com estudantes que se voluntariaram para participar, não sendo possível serem generalizados para outros alunos, possivelmente menos motivados. Apesar dessas limitações, o presente estudo contribui de forma significativa para o conhecimento sobre a relevância de implementar um programa de treinamento em EM baseado em evidências. É importante destacar os vários pontos fortes desse treinamento, que incluiu apresentações didáticas e exercícios vivenciais em um formato de workshop, realizado de acordo com as recomendações do Motivational Interviewing Network of Trainers (MINT).

Nossos resultados sugerem que receber treinamento em EM pode melhorar a confiança dos alunos de diferentes cursos da saúde para falar com os pacientes sobre uma mudança de comportamento. Mais do que isso, os achados deste estudo indicam que os estudantes, mesmo com um treinamento de oito horas, podem alcançar melhoras significativas na habilidade para utilizar a EM durante a graduação, o que pode melhorar seu desempenho no atendimento do paciente, tanto durante a vida acadêmica, quanto profissional.

Segundo White, Gazewood e Mounsey (2007), a Faculdade de Medicina da Universidade da Virginia introduziu na grade curricular desenvolver as habilidades da EM com os alunos do primeiro e terceiro ano. Os estudantes daquela amostra, assim como os da nossa, relataram perceber a necessidade de ter as habilidades da EM e um forte senso da importância e confiança na sua capacidade de utilizar a EM. Outro estudo (Decker \& Martino, 2013) também observou que, após o treinamento, os participantes obtiveram resultados positivos, com aumento significativo na confiança e interesse em utilizar a EM.

Os achados deste estudo, tomados em conjunto, têm implicações importantes para a divulgação da relevância de oferecer formação em EM para os diferentes profissionais da saúde, ainda na graduação. Apesar de estarmos satisfeitos com os resultados do estudo, é evidente que um treinamento de oito horas não é suficiente para garantir que os alunos sejam proficientes em EM. Um treinamento avançando, somado à supervisão de casos, será necessário para assegurar que essas competências estão totalmente desenvolvidas. 
Ainda, nossos dados sugerem que a metodologia empregada no treinamento foi razoavelmente eficaz, pelo menos para esse grupo de estudantes. Uma explicação alternativa de nossos resultados é que um treinamento mais intensivo possa proporcionar um aumento mais significativo dos níveis de habilidades básicas da EM. Por fim, este foi, até onde sabemos, o primeiro estudo que avaliou um treinamento das habilidades básicas da EM para estudantes de graduação de diferentes cursos da saúde no Brasil.

\section{REFERÊNCIAS}

Bell, K., \& Cole, B. A. (2008). Improving medical students' success in promoting health behavior change: a curriculum evaluation. Journal of General Internal Medicine, 23(9), 1503-1506.

Cohen, J. (1998). Statistical power analysis for the behavioral sciences $\left(2^{\text {nd }}\right.$. ed. $)$. New Jersey: Lawrence Erlbaum.

Daeppen, J. B., Fortini, C., Bertholet, N., Bonvin, R., Berney, A., Michaud, P.A., \& Gaume, J. (2012). Training medical students to conduct motivational interviewing: a randomized controlled trial. Patient Education and Counseling, 87(3), 313-318.

Decker, S. E., \& Martino, S. (2013). Unintended effects of training on clinicians' interest, confidence, and commitment in using motivational interviewing. Drug and Alcohol Dependence, 132(3), 681-687.

Dray, J., \& Wade, T. D. (2012). Is the transtheoretical model and motivational interviewing approach applicable to the treatment of eating disorders? A review. Clinical Psychological Review, 32(6), 558-565. doi:10.1016/j.cpr.2012.06.005

Lundahl, B., \& Burke, B. L. (2009). The effectiveness and applicability of motivational interviewing: A practice-friendly review of four meta-analyses. Journal of Clinical Psychology: In Session, 65(11), 1232-1245.

Madson, M. B., Loignon, A. C., \& Lane, C. (2009). Training in motivational interviewing: a systematic review. Journal of Substance Abuse Treatment, 36(1), 101-109.

Miller, W. R., Hedrick, K. E., \& Orlofsky, D.R. (1991). The Helpful Responses Questionnaire: A procedure for measuring therapeutic empathy. Journal of Clinical Psychology, 47(3), 444-448.
Miller, W. R., \& Rollnick, S. (2002). Motivational interviewing: Preparing people for change (2 ${ }^{\text {nd }}$.ed.). New York: Guilford Press.

Miller, W. R., Moyers, T. B., Ernst, D., \& Amrhein, P. (2008). Manual for the Motivational Interviewing Skill Code (MISC) Version 2.1. Recuperado de http://casaa.unm.edu/download/ misc.pdf.

Miller, W. R., \& Rollnick, S. (2009). Ten things that motivational interviewing is not. Behavioural and Cognitive Psychotherapy, 37(2), 129-140.

Miller, W., \& Rollnick, S. (2013). Motivational Interviewing: Helping people change ( $3^{\text {rd }}$.ed.). Guilford Press: New York.

Moyers, T. B., Martin, T., Manuel, J. K., Miller, W. R., \& Ernst, D. (2007). Revised global scales: Motivational Interviewing Treatment Integrity 3.0 (MITI 3.0). Recuperado de http://casaa. unm.edu/download/miti3.pdf.

Rollnick, S., Miller, W.R., \& Butler, C. (2009). Motivational interviewing in health care: Helping patients change behavior. Guilford Press: New York.

Söderlund, L. L., Madson, M. B., Rubak, S., \& Nilsen, P. (2011). A systematic review of motivational interviewing training for general health care practitioners. Patient Education and Counseling, 84(1), 16-26.

White, L. L., Gazewood, J. D., \& Mousey, A. L. (2007). Teaching students behavior change skills: Description and assessm. Medical Teacher, 29(4), 67-71. 